\section{Variable wavelength- spacing tunable filter using polarization differential delay line}

\author{
Seunghwan Chung \\ Byoungho Lee, FELLOW SPIE \\ Seoul National University \\ School of Electrical Engineering \\ Seoul 151-744, Korea \\ E-mail: byoungho@snu.ac.kr
}

\begin{abstract}
A tunable filter that can be applied for dispersion compensation, a multiwavelength laser source, or a channel transmitter/attenuator is proposed. Channel spacing and dispersion can be controlled simultaneously by using a motorized polarization differential delay line and a variable coupler in the loop mirror. Due to its periodic characteristic, chromatic dispersion of multiple channels can be compensated all at once. (C) 2005 Society of Photo-Optical Instrumentation Engineers. [DOI: 10.1117/1.1849752]
\end{abstract}

Subject terms: optical fiber filters; optical fiber dispersion; phase response; delay line.

Paper L040351 received Jun. 8, 2004; revised manuscript received Oct. 20, 2004; accepted for publication Oct. 25, 2004; appeared online Nov. 2, 2004; published online Feb. 1, 2005.

\section{Introduction}

With the constant increase in traffic demand and the advent of reconfigurable optical networks, a tunable optical comb filter has been demonstrated to be useful in many different areas of optical communication ${ }^{1-4}$ Especially, an optical all-pass filter using a ring resonator is worth noticing. However, it is better for the channel spacing of the filter and dispersion to be controlled simultaneously by various conditions. In this work, we propose a new simple filter for multichannel dispersion compensation using a motorized polarization differential delay line (DDL). It is possible to change easily the channel spacing with simple control electronics. In addition, we can control the group-delay characteristics of the proposed device by changing the powercoupling ratio of the variable coupler.

\section{System Configuration}

Figure 1(a) shows the schematic diagram of the proposed variable channel-spacing dispersion compensation filter. The loop mirror consists of a polarization controller (PC), a variable coupler (Fiberpro, TC1410, Daejeon, Korea), and a DDL (OZ Optics, DDL-650, Carp, Ontario, Canada). Control of the splitting ratio in the variable coupler is achieved by adjusting the relative lateral positions of the mated fibers with the help of micrometers. DDL is the device that splits the light within a fiber into orthogonal polarizations, and then actively varies the time that one polarization travels

0091-3286/2005/\$22.00 @ 2005 SPIE compared to the other polarization before combining the two polarizations together again. The PC in the loop acts as a polarization state rotator for both clockwise and counterclockwise beams. It changes a fast-axis beam to a slow-axis beam and vice versa. These two beams recombine and have phase differences proportional to the polarization differential delay. The wavelength spacing of the peaks is as follows ${ }^{2}$ :

$\Delta \lambda=\frac{\lambda^{2}}{c \Delta t}$,

where $c$ is the speed of light in the air, $\Delta t$ is the difference in the delay between the two polarizations, and $\lambda$ is the wavelength of the light. The distance difference in DDL is controlled by a reflector on a moving stage. By altering $\Delta t$, we can vary wavelength spacing of the peak, that is, channel spacing of the filter. Figure 1(b) shows the measured spectra when $\Delta t$ is changed. The larger the value of $\Delta t$ is, the smaller the channel spacing is. The resolution of the polarization DDL was $0.0017 \mathrm{ps}$ and the delay range was $\pm 50 \mathrm{ps}$. This shows us that the proposed system is able to change the channel spacing nearly continuously.

The phase response of the output beam can be obtained from the following:

$U_{o}=U_{i}\left[t^{2} \exp \left(j \Phi_{f}\right)+r^{2} \exp \left(j \Phi_{s}\right)\right]$,

where $U_{o}$ and $U_{i}$ are the output light at the transmission end and the incident light of the filter; $t$ and $r$ are throughand cross-amplitude transmissions of the tunable coupler, which are designated by $t=\sqrt{1-\kappa}, r=j \sqrt{\kappa}$, respectively, where $\kappa$ is the power-coupling ratio; and $\Phi_{f}$ and $\Phi_{s}$ are the phases of the fast and slow axes light, which are designated by $\Phi_{f}=n_{f} L \omega / c, \Phi_{s}=n_{s} L \omega / c$. Here, $L$ is the physical length of DDL, and $n_{s} L$ and $n_{f} L$ are the optical path lengths for lights propagating with the slow and fast polarizations of the DDL, respectively. In Eq. (2), we assume that the input light polarization is matched to the slow axis of the DDL. The PC rotates the polarization by $90 \mathrm{deg}$. The wavelength-dependent phase difference between the two orthogonal polarization axes of the DDL produces a sinusoidal wavelength-dependent filter transmission function, as given by

$\left|\frac{U_{o}(\lambda)}{U_{i}}\right|^{2}=1-2 \kappa+2 \kappa^{2}-2\left(\kappa-\kappa^{2}\right) \cos \left(\Phi_{f}-\Phi_{s}\right)$.

From Eq. (2), we can obtain the relative group delay. It can be given by the following equation:

$$
\begin{aligned}
\tau(\omega) & =-\frac{d \Phi_{t}(\omega)}{d \omega} \\
& =-\left(\frac{n_{f} L}{c}\right) \frac{1+p^{2}\left(\frac{n_{s}}{n_{f}}\right)+p\left(1+\frac{n_{s}}{n_{f}}\right) \cos (\omega \Delta t)}{1+p^{2}+2 p \cos (\omega \Delta t)},
\end{aligned}
$$

where $\Phi_{t}(\omega)$ is the phase of Eq. (2), i.e., $\Phi_{t}(\omega)$ $=\arg \left(U_{o} / U_{i}\right)$ and $p=r^{2} / t^{2}=-\kappa /(1-\kappa)$.

The predicted group delay results are compared with the measured data in Fig. 2 and good agreement is reported. 


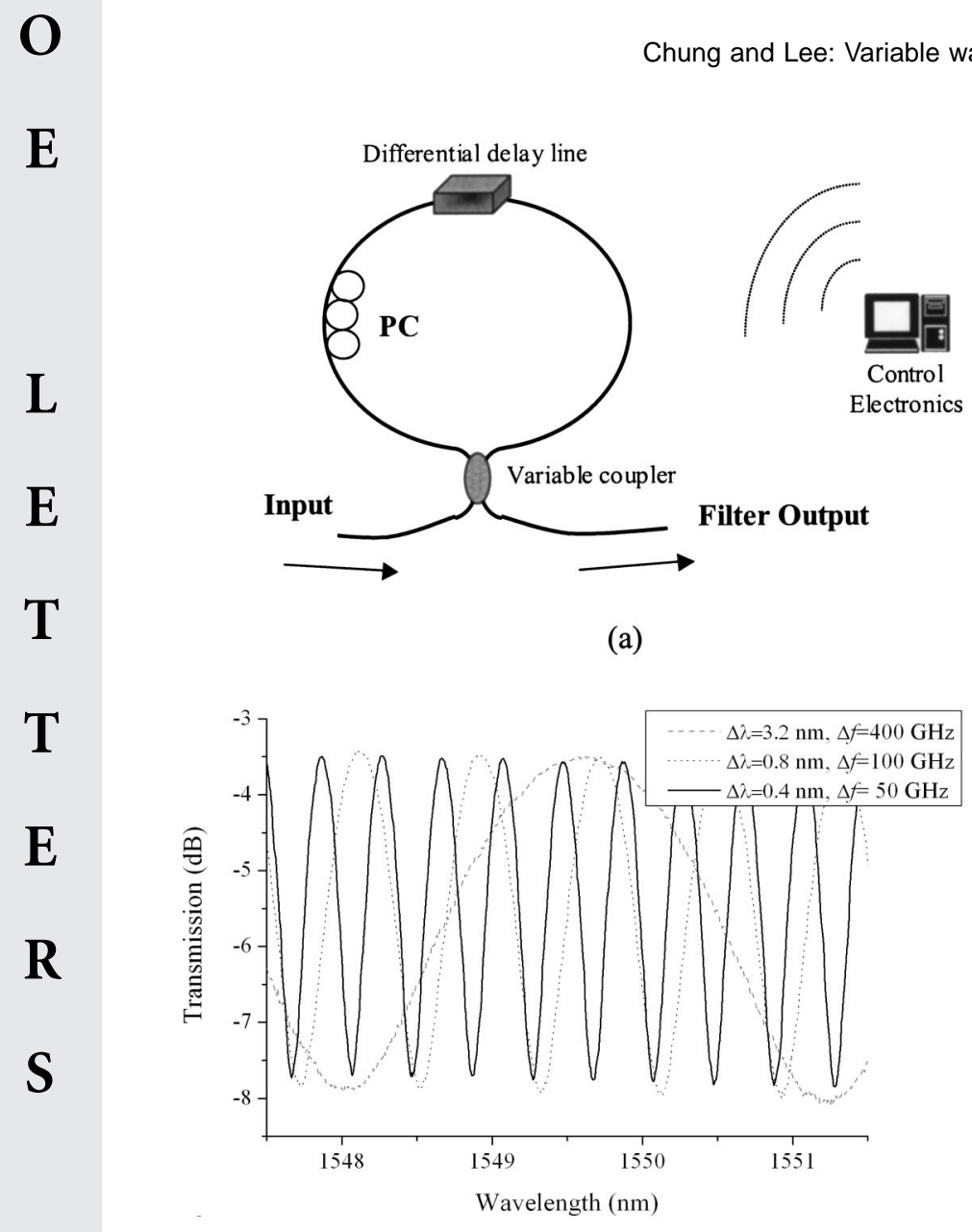

(b)

Fig. 1 (a) Basic configuration of variable dispersion equalizer and (b) measured spectrum of the filter output when $\Delta t$ is changed. ( $\Delta t$ is $2.5,10$, and 20 ps for $\Delta f=400,100$, and $50 \mathrm{GHz}$, respectively.)

Figure 2(a) shows the calculated relative group delay characteristic when $\Delta t$ is varying as 5,10 , and $20 \mathrm{ps}$. Figure 2(b) shows the measured characteristics of the filter when $\kappa$ is 0.6 .

\section{Discussion and Conclusions}

We propose a variable channel-spacing tunable filter for dispersion compensation and demonstrate its feasibility by experiment. In the proposed method, the channel spacing and the dispersion can be varied with the change of polarization delay and the coupling constant by a simple tuning method. We can vary the channel spacing nearly continuously with the use of DDL controlled by a computer. The tuning speed is mainly dependent on a control interface unit and the moving stage's speed in a DDL. The maximum speed can be up to a few megahertz or more. The temperature stability of this system is very good compared to other systems using interferometry or polarization maintaining fibers. These features can make it more useful and compatible to various environments. And in the need of greater design flexibility, the architecture can be easily extended to higher orders by cascading many rings. Because the PC used in the loop does not change the polarization state completely, and the power-coupling coefficient in our variable

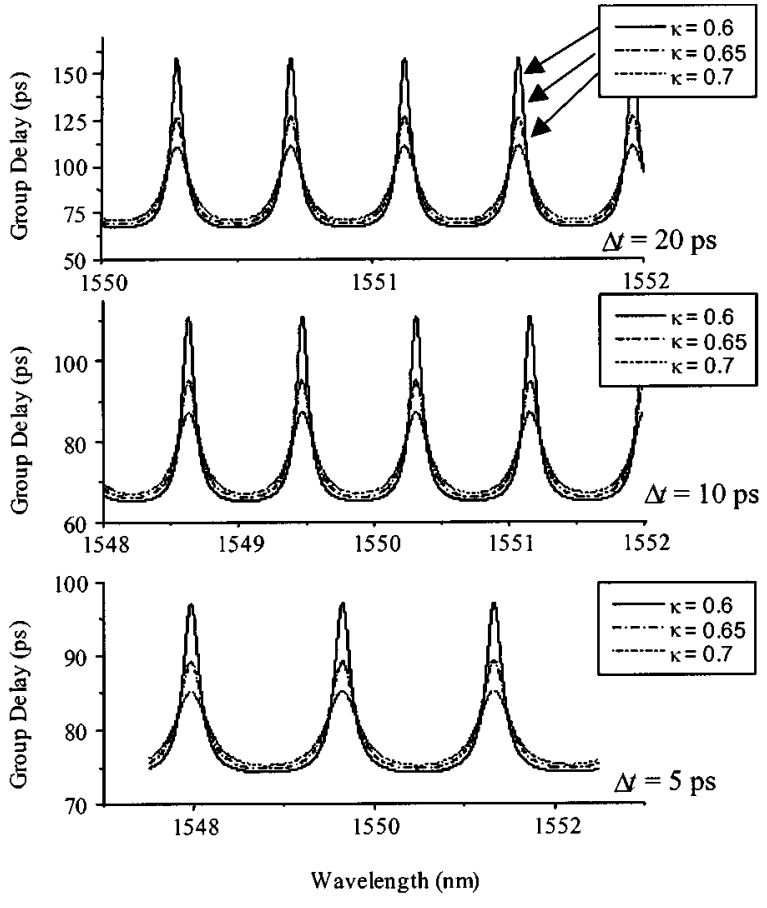

(a)

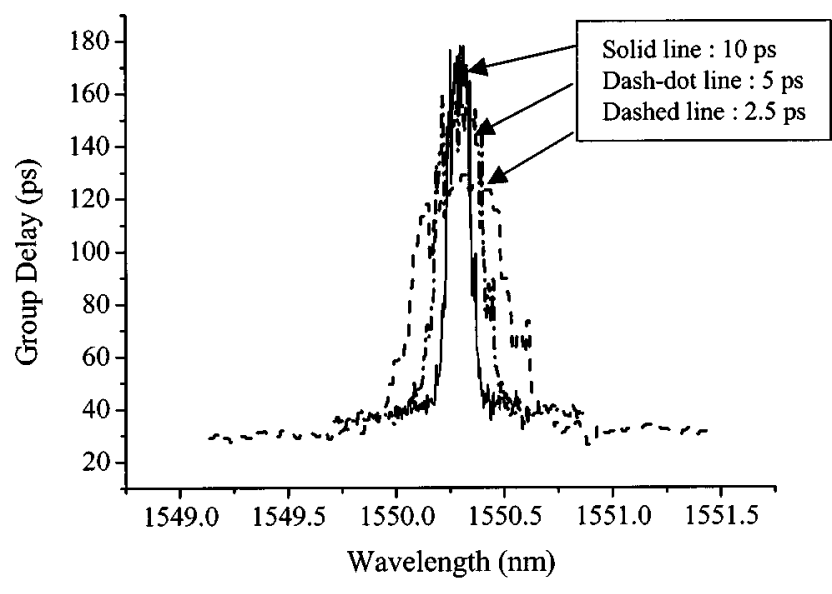

(b)

Fig. 2 (a) Calculated relative group delay of a basic configuration filter. (b) Measured relative group delay when the differential delay is $2.5,5$, and $10 \mathrm{ps}$ and $\kappa$ is 0.6 . The dispersion is $514.8,192.9$, and $86.8 \mathrm{ps} / \mathrm{nm}$, respectively.

coupler has some errors, there is difference between the calculated and measured results.

\section{References}

1. R. M. Sova, C. S. Kim, and J. U. Kang, "Tunable all-fiber birefringence comb filters," Tech. Dig. OFC'02, pp. 698-699 (2002).

2. C. Madsen and G. Lenz, "Optical all-pass filters for phase design with applications for dispersion compensation," IEEE Photonics Technol. Lett. 10(7), 994-996 (1998).

3. C. Madsen, G. Lenz, A. J. Bruce, M. A. Cappuzzo, L. T. Gomez, and R. E. Scotti, "Integrated all-pass filters for tunable dispersion and dispersion slope compensation," IEEE Photonics Technol. Lett. 11(12), 1623-1625 (1999).

4. S. Chung, B. A. Yu, and B. Lee, "Phase response design of a polarization maintaining fiber loop mirror for dispersion compensation," IEEE Photonics Technol. Lett. 15(5), 715-717 (2003). 